This is the accepted manuscript made available via CHORUS. The article has been published as:

Testing spin-flip scattering as a possible mechanism of ultrafast demagnetization in ordered magnetic alloys Stefan Günther, Carlo Spezzani, Roberta Ciprian, Cesare Grazioli, Barbara Ressel, Marcello Coreno, Luca Poletto, Paolo Miotti, Maurizio Sacchi, Giancarlo Panaccione, Vojtěch Uhlíř, Eric E. Fullerton, Giovanni De Ninno, and Christian H. Back

Phys. Rev. B 90, 180407 - Published 21 November 2014 DOI: 10.1103/PhysRevB.90.180407 


\title{
Testing spin-flip scattering as a possible mechanism of ultrafast demagnetization in ordered magnetic FeRh alloy
}

\author{
Stefan Günther ${ }^{1}$, Carlo Spezzani ${ }^{2}$, Roberta Ciprian $^{2}$, Cesare Grazioli ${ }^{2,3}$, Barbara Ressel ${ }^{3}$, \\ Marcello Coreno ${ }^{2,4}$, Luca Poletto ${ }^{5}$, Paolo Miotti ${ }^{5}$, Maurizio Sacchi ${ }^{6}$, Giancarlo Panaccione ${ }^{2}$, \\ Vojtěch Uhlíŕr ${ }^{7}$ Eric Fullerton ${ }^{7}$, Giovanni De Ninno ${ }^{3,2}$, and Christian H. Back ${ }^{1}$ \\ 1 University of Regensburg, Germany \\ 2 Elettra Sincrotrone Trieste, Trieste, Italy \\ 3 Laboratory of Quantum Optics, University of Nova Gorica, Nova Gorica, Slovenia \\ ${ }^{4}$ Institute of Inorganic Methodologies and Plasmas (CNR-IMIP), Montelibretti, Roma, Italy \\ ${ }^{5}$ Institute of Photonics and Nanotechnologies (CNR-IFN), Padova, Italy \\ 6 CNRS, Paris, France and \\ 7 UCSD, San Diego, USA
}

\begin{abstract}
We use element resolved IR-pump/XUV-probe experiments to disentangle the ultrafast inter-play of the magnetic sublattices of an ordered crystalline magnetic alloy. As a paradigmatic example, we investigate the case of the FeRh alloy, which shows a delayed response for the different components. Furthermore, a detailed time resolved magneto-optic study shows that the data can be analyzed by only assuming Elliot-Yafet-like scattering, as the underlying mechanism for ultrafast demagnetization, resulting in an unexpected non-monotonic dependence of the spin-flip rate, as a function of quenching.
\end{abstract}


In recent years a substantial experimental effort has been devoted to the study of ultrafast demagnetization and all-optical switching of ferro- and ferrimagnetic alloys. Understanding these phenomena is of interest for heat assisted magnetic recording and all-optical magnetic recording [1]; however, unraveling the microscopic mechanisms behind the observed dynamic response has remained a formidable task. Several theories have been put forward claiming to be able to explain ultrafast demagnetization in general [2-9] and in particular the response of multi-sublattice systems where two or more magnetic elements form a ferroor ferrimagnetically coupled ordered system [10-12]. In these systems, a crucial question is how different elements in an alloy interact on a ultrashort timescale close to the timescale corresponding to the exchange interaction (10-100 fs).

Element specific probes [13-15] as well as systematic studies using more conventional time resolved techniques [16] on ultrashort time scales have had a tremendous share in the advancement of knowledge in this particular field. For example, for ferrimagnetic 3dtransition metal / 4f-rare earth alloys (3d-4f) such as CoFeGd used for all-optical switching, that a transient ferromagnetic state exists in the process of ultrafast magnetization reversal [17]. In spite of their strong antiferromagnetic exchange coupling, the individual components of these systems show independent demagnetization dynamics characterized by different demagnetization times. Furthermore, it has been shown recently, that highly ordered 3d-4f multilayer systems can also be used for all-optical switching [18].

Theoretical models based on a description of different anti- or ferromagnetically coupled sublattices with distinct coupling constants have been put forward to explain the elementspecific behavior of these systems [10-12]. However, no consensus on the underlying microscopic demagnetization process in multi-sublattice systems exists.

Interestingly, there seems to be no experimental consensus on the demagnetization dynamics even for ferromagnetically coupled multi-sublattice systems such as simple 3dtransition metal alloys. In the case of $\mathrm{NiFe}$, for instance, two different element specific experimental probes performed at the $2 \mathrm{p}$ and $3 \mathrm{p}$ resonances (time resolved magneto-optics at the L-edges or M-edges) gave contrasting results, with Ni demagnetizing either faster [19] or slower [20] than Fe. There is thus the need to study and unravel the demagnetization behavior of multi-sublattice systems in particular in view of understanding the possibly technologically relevant all-optical switching mechanism. One experimental shortcoming that complicates a direct comparison to theoretical predictions (in particular predictions 
based on the electronic band structure [21]), is the fact that so far only disordered or polycrystalline alloys have been studied experimentally. Concentrating on theoretically more easily accessible ordered crystalline alloys, can help understanding the origin of the different demagnetization dynamics of the alloy components, by comparing experiments to theoretical calculations. In this letter, we concentrate on an ordered multi-sublattice system, the ordered alloy, FeRh, a single-crystalline material with a well-known electronic band structure [22]. This ordered alloy undergoes a first order phase transition from an antiferromagnetically ordered phase to a ferromagnetically ordered phase at temperatures slightly above room temperature. The second order phase transition to the paramagnetic phase occurs around 660 Kelvin. Quite a few time resolved experiments have addressed the fundamental question of the ultimate time scale for the generation of magnetic order in this particular system when driven through the first order phase transition. However, only little is known about the demagnetization dynamics of the FeRh system when excited by powerful laser pulses in its ferromagnetic state [23] which is at the heart of this article. Two experimental methods based on time-resolved magneto-optics are exploited. We use the time resolved magneto-optic Kerr effect (TR-MOKE) in the visible to IR range as well as in the XUV (TR-XUV-MOKE) domain. Using high harmonic generation (HHG), element specific information can be accessed at the M/N-edges. Our element specific experiments indicate that the demagnetization of Fe is delayed with respect to $\mathrm{Rh}$, and has a slightly shorter demagnetization time. This finding is supported by the microscopic three temperature model (M3TM) [2] which we employ to model our data. Additional detailed TR-MOKE experiments, presented in the following, allow us to test the M3TM model more accurately and reveal a clear dependence of the spin-flip rate on the exciting laser fluence, that cannot be accounted for within a rigid band approach, where the spin-flip rate stays constant.

For the experiments we use epitaxially grown FeRh films with a thickness of $50 \mathrm{~nm}$ deposited onto a $\mathrm{MgO}(001)$ substrate by magnetron sputtering from a single equiatomic target. The FeRh films grow epitaxially with (001) orientation. The high quality films show a typical temperature hysteresis of $20 \mathrm{~K}$ with the first order phase transition from the antiferromagnetic to the ferromagnetic phase appearing around $360 \mathrm{~K}$. The Curie temperature is reached at $660 \mathrm{~K}$.

In order to study the element selective ultrafast demagnetization dynamics of FeRh, we have implemented a TR-XUV-MOKE experiment at the novel High Harmonic Generation 


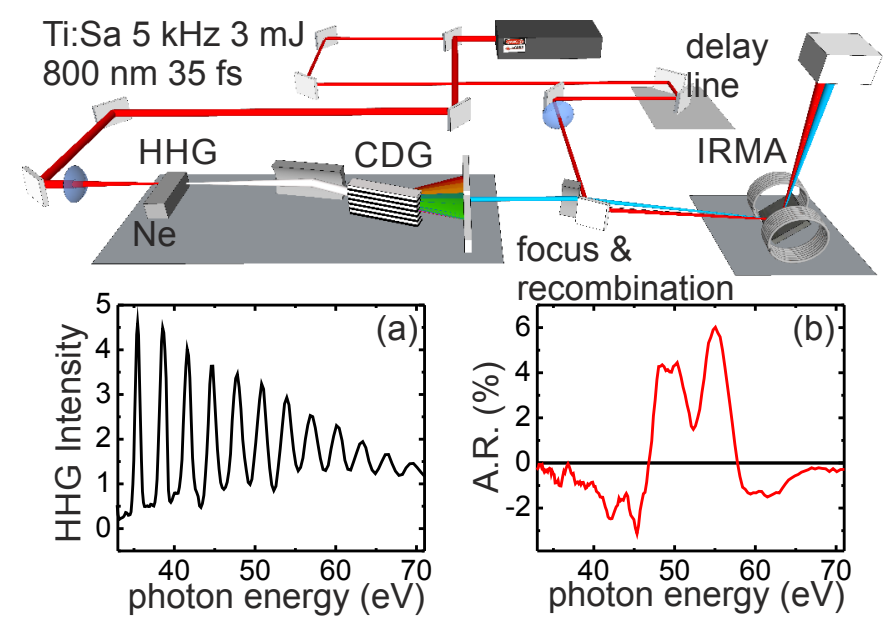

FIG. 1. Sketch of the CITIUS HHG light source. The $3 \mathrm{~mJ} /$ pulse Ti:Sapphire laser output is split in two branches: $2 \mathrm{~mJ} /$ pulse are used for HHG and $1 \mathrm{~mJ} /$ pulse for sample excitation (IR pump). The proper harmonic is selected by means of a time preserving monochromator based on conical diffraction gratings (CDG) and refocalized onto the sample. The IR pump is recombined with the HHG light in the experimental chamber IRMA on the sample in an almost collinear geometry. a) The higher harmonics spectrum from Ne after the monochromator captured with a photodiode and b) the normalized magnetic asymmetry ratio (A.R.), namely the difference of both field directions divided by their sum, for the T-MOKE like configuration in the IRMA setup.

(HHG) beamline CITIUS [24](see Fig. 1). This light source is based on standard HHG in noble gases $[25,26]$ and makes use of a high power Ti:Sapphire ultrashort laser amplifier delivering $3 \mathrm{~mJ}$ pulses at $800 \mathrm{~nm}$ (repetition rate $5 \mathrm{kHz}$ ) with a typical pulse duration of 35 fs [24]. A beam splitter is used to supply HHG with two thirds of the amplifier output. The remaining fraction is used for the excitation of the system using a variable time delay. High harmonics up to order (H)51 of the fundamental wavelength with a linear vertical polarization are obtained.

A spectral selection of the light sent to the sample is made by means of a pulse-duration preserving monochromator [27, 28]. The IR pump (spot size about $500 \mu \mathrm{m}$ FWHM) and XUV probe beam (spot size about $200 \mu \mathrm{m}$ FWHM) are recombined on the sample in an almost collinear geometry in the IRMA reflectometer featuring a vertical scattering plane [29]. The maximum temporal inaccuracy by changing the XUV wavelength, due to movements in the monochromator, is estimated to be below 5 fs for the chosen harmonics. A 
deatiled technical description of the HHG-beamline can be found in Ref. [24]. The scattered light is detected using a photodiode mounted on the detector arm, shielded with a $200 \mathrm{~nm}$ thick Al filter to block the IR pump. In the T-MOKE-like configuration using p-polarized XUV light, one is sensitive to the magnetization component perpendicular to the scattering plane. Choosing the incoming/scattering angle close to the Brewster condition (about $45 \mathrm{deg}$ at $54 \mathrm{eV}$ ), the magnetization contrast is maximized with respect to the strongly suppressed non-magnetic scattered background [30, 31]. By varying the photon energy, the harmonics for the Fe M-(H35, i.e. $54 \mathrm{eV})$ and Rh N-edges (H31/H33, i.e. $48 / 51 \mathrm{eV})$ can be identified and the signals of the two chosen harmonics (H31 and H35) are clearly separated, see Fig. 1b). To avoid longterm drifts, the magnetic asymmetry for Fe and Rh is measured via field reversal at every time delay between IR pump and XUV probe and then multiple demagnetization traces are averaged. In the following $t_{0}$ is defined as the average value for the beginning of the initial drop of the magnetization of Fe and Rh.

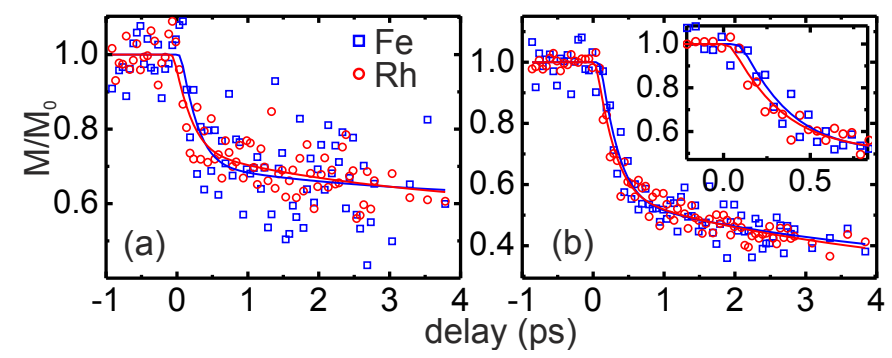

FIG. 2. Element resolved demagnetization dynamics of FeRh (Fe: blue open squares, Rh: red open circles) obtained by TR-XUV-TMOKE. The reduced magnetization $\left(M / M_{0}\right)$ after excitation with a) $11 \mathrm{~mJ} / \mathrm{cm}^{2}$ and b) $16 \mathrm{~mJ} / \mathrm{cm}^{2}$. The inset in b) shows a zoom to the initial drop of the signal. Fe demagnetization is delayed with respect to Rh by approximately 60 fs.

In addition, two color TR-MOKE experiments are performed. For these measurements we use a regenerative amplified Ti:Sapphire laser, which provides up to $4 \mu \mathrm{J}$ pulses with a pulse length below $50 \mathrm{fs}$ and a repetition rate which is adjustable between $70-250 \mathrm{kHz}$. We use the fundamental IR pulse $(800 \mathrm{~nm})$ for pumping and the second harmonic visible pulse (400 nm) for probing the magnetic state. The temperature is kept constant at $400 \mathrm{~K}$ for all measurements.

Two typical TR-XUV-MOKE demagnetization traces in the fully ferromagnetic phase (which is verified by recording a static temperature hysteresis loop) are plotted for two 
different fluences in Fig. 2a,b). The fit (solid line) is performed using the M3TM with initial parameters obtained by TR-MOKE on the same sample and will be further addressed below. A small but non negligible shift in time zero of the two different element specific demagnetization traces appears within the experimental accuracy in all measurements. We find that $\mathrm{Fe}$ is delayed with respect to $\mathrm{Rh}$ by approximately 60 fs and the evolution of the shift as a function of pump fluence (or respective quenching) is summarized in Fig. 4c). Moreover we find that the demagnetization time of Fe is slightly shorter (by about $25 \mathrm{fs}$ ) than the one of Rh. We would like to note, that the timeshift between Fe and Rh is confirmed by a fully statistical analysis of the data.

To shed further light on the experimental findings and to test the validity the use of the M3TM, we perform detailed TR-MOKE experiments at lower pump fluences with higher signal to noise ratio with respect to XUV-MOKE. We observe partial demagnetization of the FeRh films with an initial fast drop of the magnetization followed by a decay with a much lower rate before the system starts to relax back to its equilibrium value, see Fig 3, which - in the classification of Koopmans et al. [2] - is called type II demagnetization behavior with a transition to type I (no slow decay) for the lowest fluence. When increasing the incident fluence the quenching $\left(1-M / M_{0}\right)$ increases and the type II behavior becomes more pronounced.

Since for type II behavior the definition of the time scale for the initial fast drop of the magnetization is somewhat arbitrary, in this paper the demagnetization time for type II demagnetization is defined as the time where the system has completed 1 /e of its demagnetization with respect to its value at 1 ps after time zero $t_{0}$. The quenching is consequently defined as the value at 1 ps, see Fig 3 .

In the following we will relate our findings to existing models for demagnetization in multisublattice systems $[10,12]$. Not all existing models can be applied to our experimental system since typical input parameters for these models are related to the "pure" phase, in our case pure Fe or pure Rh. Even though the magnetic moments of Fe and Rh in the ferromagnetic phase are known $[32,33]$, the moment of $\mathrm{Rh}$ in the pure phase vanishes and consequently a demagnetization time can not be determined. In systems where a 'pure' phase exists like in Fe-Ni alloys it was shown that the difference in $t_{0}$ and $\tau$ is related to the strength of exchange coupling between the involved sublattices [20].

Note that the experimentally observed shift in $t_{0}$ can be explained by using Elliot-Yafet- 


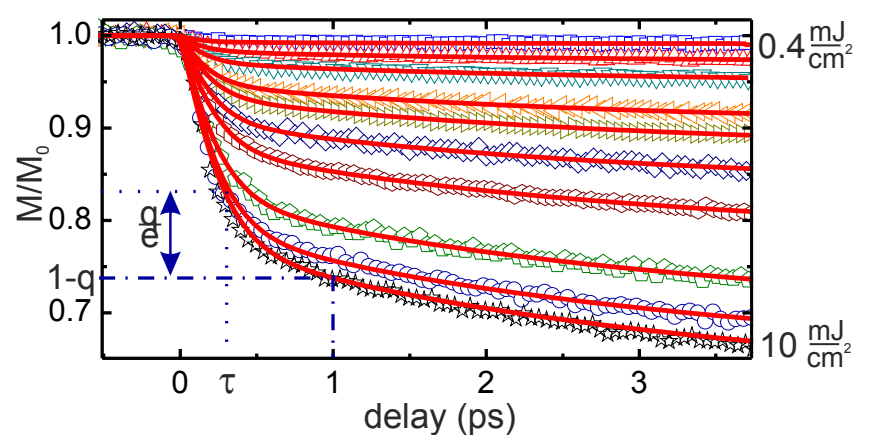

FIG. 3. TR-MOKE demagnetization traces of FeRh for various fluences $\left(0.4-10 \mathrm{~mJ} / \mathrm{cm}^{2}\right)$. The solid line (red) is a fit using the M3TM-model, where only the spin-flip rate and the excitation strength is allowed to vary. The demagnetization time $\tau$ (dotted line) referenced to the quenching $\mathrm{q}$ (defined as the loss of magnetization at $1 \mathrm{ps}$, dashed-dotted line) is visualized for the trace with the highest fluence $\left(10 \mathrm{~mJ} / \mathrm{cm}^{2}\right)$.

type spin-flip scattering as a key mechanism and therefore our data can be analyzed within the M3TM model $[2,12]$. We would like to note that other theories relying on Elliott-Yafettype spin flip scattering (with and without dynamic feed-back mechanism) [21, 34] may be able to explain the data as well. In our case we refrain to the simple in a rigid band like approach M3TM model which in principle can be applied without the need of band structure calculations. It is exactly the rigid band approach, that will be under scrutiny in the following. We would also like to note that we neglect super diffusive transport, which is probably an additional source for the loss of magnetic contrast [4], but is not discussed in this paper, because its a thin single-domain film deposited on an insulator.

The rate equations of the M3TM read:

$$
\begin{gathered}
C_{e}\left[T_{e}\right] \frac{d T_{e}}{d t}=g_{e p}\left(T_{p}-T_{e}\right)+G\left(t_{0}, T_{e, \max }\right) \\
C_{p} \frac{d T_{p}}{d t}=g_{e p}\left(T_{e}-T_{p}\right) \\
\frac{d m}{d t}=R\left[g_{e p}, a_{s f}\right] m \frac{T_{p}}{T_{C}}\left(1-m \operatorname{coth}\left(\frac{m T_{c}}{T_{e}}\right)\right)
\end{gathered}
$$

where $T_{e}, T_{p}$, denote the electronic and the phononic temperatures, $G\left(t_{0}, T_{e, \max }\right)$ the Gaussian excitation due to the pump pulse at time zero $\left(t_{0}\right)$ with a maximum electronic temperature $T_{e, \max }, m=\frac{M}{M_{S}}$, the magnetization relative to its expected zero temperature value, $T_{C}$ the Curie temperature, $g_{e p}$ the electron-phonon coupling and $C_{p}$ and $C_{e}[T-e]$ the 
specific heat of the phononic system and the electronic system (in linear approximation with a proportionality constant $\gamma) . C_{p}$ is treated as a constant value for the whole covered temperature range, in agreement with static measurements [35]. $R=\left(8 a_{s f} g_{e p} k_{B} T_{C}^{2}\right) V_{a t} /\left(\frac{\mu_{a t}}{\mu_{B}} E_{D}^{2}\right)$ is a material specific parameter, where $\frac{\mu_{a t}}{\mu_{B}}$ is the ratio of the atomic magnetic moment and the Bohr magnetron, $V_{a t}$ the atomic volume, $E_{D}$ the Debye energy and $a_{s f}$ the spin-flip parameter, which is typically assumed constant within a rigid band approach. A cooling mechanism by heat diffusion to the substrate was found to be negligible for the behavior during the first 4 ps and is therefore neglected.

To obtain values for those variables that can not be determined from static experiments, but are necessary to model the dynamic data (e.g. $\left.a_{s f}, \gamma, g_{e p}\right)$, the transient optical response for all fluences is fitted by eq. 1-2. With the assumption that the optical response is a good approximation for the electronic temperature, the electron specific heat shows a linear dependence and thus validates $C_{e}=\gamma T_{e}$. By additionally assuming $T_{p} \ll T_{e}$, $g_{e p}$ can be determined on short timescales. Note that $g e_{e p}$ and $a_{s f}$ can in principle be calculated using ab-initio methods at least in the limit $T_{e} \rightarrow 0$.

When fitting the traces for all fluences, it is possible to keep only 3 modeling parameters free, namely the $a_{s f}, T_{e, \max }$ and $t_{0}$. Before fitting the experimental data with the Levenberg-Marquardt algorithm, the solution for $M / M_{0}$ of the M3TM model is convoluted with a Gaussian pulse to account for the influence of the finite pulse length and the shape of the probing pulse.

Allowing only the spin-flip rate to vary - which strongly influences the demagnetization time and the quenching - the variation in the demagnetization time is also reflected by a drastic change in the spin-flip rate $a_{s f}$ (Fig $\left.4 \mathrm{a} / \mathrm{b}\right)$. The demagnetization time $\tau$ shows a drastic change for low fluences while for intermediate and high fluences only small variations are observed. In the low fluence range the fast increase of the demagnetization time is caused by the steep decrease of the spin flip-rate $a_{s f}$. The non linear behavior of $a_{s f}$ extracted from the M3TM is a clear indication that the electronic band structure changes and can not be treated as being rigid with increasing fluence [36], however the trend and the clearly visible minimum around 10 percent quenching still remain unclear.

We believe that the non-monotonic behavior of $a_{s f}$ is aclear indication of the oversimplification within the M3TM (use of rigid bands). We would also like to emphasize that the subtle effects observed here might disappear when polycrystalline or amorphous materials 
are investigated opposed to the single crystalline material studied here due to the loss of a well defined electronic band structure. However, even for a single crystalline material detailed investigations of the dynamic band structure on short time scales are a formidable task and beyond the scope of this paper. Nevertheless the value for the spin-flip rate is always in the range of calculated values [22] for the spin-mixing parameter $\left\langle b^{2}\right\rangle$, with $a_{s f}=p\left\langle b^{2}\right\rangle$ and $p$ ranging from 1.8 to 4 . Furthermore $\left\langle b^{2}\right\rangle$ is calculated to be smaller on the Fe site than on the Rh site, which is also qualitatively reflected in the element selective measurements (Fig 4b). Note that, it is not possible to fit the fluence dependence by only varying the excitation energy. Although $g_{e p}$ is expected not to change much in this fluence regime [37-39], the fit of the optical response reveals a different behavior (Fig 4d), and may be related to changes of $a_{s f}$ in the fit since $a_{s f}$ and $g_{e p}$ are somehow entangled. Fortunately, variations of $g_{e p}$ are found to hardly influence the signature of $a_{s f}$, neither with $g_{e p}$ as a fitting parameter, nor with fixed values for each fluence, as indicated by carefully analyzing the transient optical response (Fig 4d).

In summary, in this paper we have presented a fluence dependent demagnetization experiment for an ordered single crystalline alloy, with elemental resolution and additional information obtained by TR-MOKE. The data can be modeled using Elliot-Yafet-like scattering as a driving mechanism for ultrafast demagnetization within the framework of the M3TM. Furthermore the observed element dependent shift in $t_{0}$ can be qualitatively described within this theory. The strong hybridization between the Fe and Rh states results in a strong coupling of the Fe and Rh moments [22] and may therefore explain the relatively small differences between the demagnetization traces, in contrast to the larger differences observed in the much weaker coupled 3d-transition metals rare earth alloys [17]. However, the observed dependence of the spin-flip rate $a_{s f}$ with a clear visible minimum as a function of quenching (or fluence) cannot be understood within a rigid band approach and remains an unsolved problem awaiting further theoretical input. We would like to note here, that this dependence can only be observed when a detailed set of experimental data as the one presented here is available. Moreover, it would be interesting to test in future calculations, if e.g. the proposed dynamic feedback mechanism within an Elliot-Yafet-like scattering approach [21] allows explaining the data, or if theory has to be refined even further. We would like to emphasize that the spin-flip rate should be accessible for single crystalline ordered alloys from ab initio theories. A possible contribution from superdiffusive transport should 

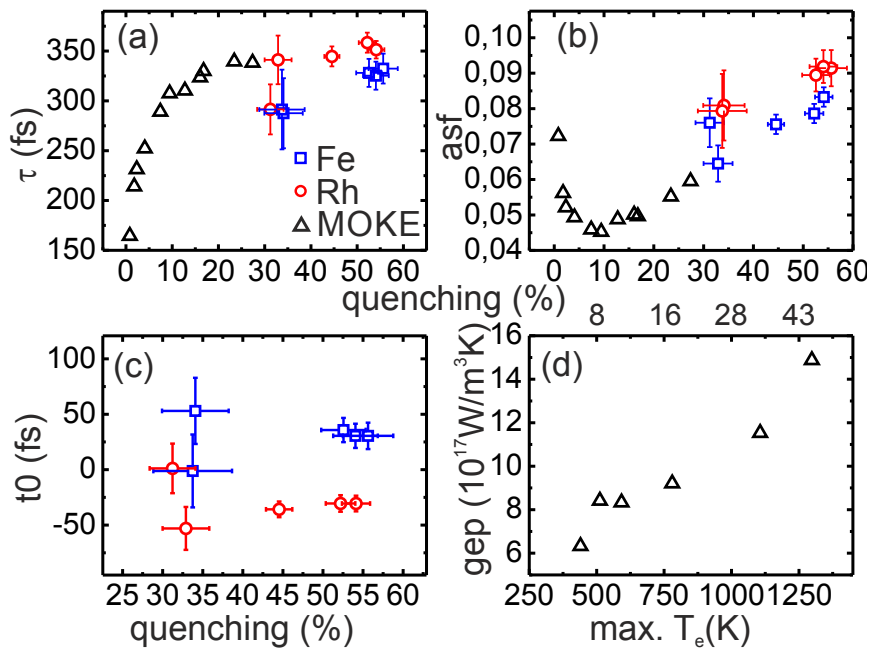

FIG. 4. Fit parameters from the M3TM for element resolved measurements, Rh (red open circles), Fe (blue open squares) and for TR-MOKE and the transient optical response (black open triangles, errorbars are within the symbol size). a) The demagnetization time $\tau$ and the b) spin-flip rate $a_{s f}$ which is a key parameter for describing the demagnetization time and the quenching versus fluence. c) The difference in $t_{0}$ for the element resolved demagnetization times shows that Fe starts delayed by about 60 fs with respect to Rh for various fluences. Errorbars for lower fluence appear bigger due to a lower signal and a nearly constant noise level in all measurements. d) The electron phonon coupling $g_{e p}$ is not constant as a function of the maximum electronic temperature or the resulting calculated quenching.

also be present, but has not been treated in this paper since the emphasis has been put on the explanation of the distinct demagnetization behavior of the two sublattices.

We would like to acknowledge Hubert Ebert for fruitful discussions. UCSD was supported under DOE-BES Award No. DE-SC0003678. The CITIUS project is funded by the program for crossborder cooperation between Italy and Slovenia 2007-2013.

[1] B. C. Stipe, T. C. Strand, C. C. Poon, H. Balamane, T. D. Boone, J. A. Katine, J.-L. Li, V. Rawat, H. Nemoto, A. Hirotsune, et al., Nature Photonics 4, 484 (2010).

[2] B. Koopmans, G. Malinowski, F. Dalla Longa, D. Steiauf, M. Fähnle, T. Roth, M. Cinchetti, and M. Aeschlimann, Nat. Mater. 9, 259 (2009).

[3] M. Krauß, T. Roth, S. Alebrand, D. Steil, M. Cinchetti, M. Aeschlimann, and H. C. Schneider, 
Phys. Rev. B 80, 180407 (2009).

[4] M. Battiato, K. Carva, and P. M. Oppeneer, Phys. Rev. Lett. 105, 027203 (2010).

[5] M. Fähnle, M. Haag, and C. Illg, JMMM 347, 45 (2013).

[6] E. Carpene, E. Mancini, C. Dallera, M. Brenna, E. Puppin, and S. De Silvestri, Phys. Rev. B 78, 174422 (2008).

[7] G. P. Zhang and W. Hübner, Phys. Rev. Lett. 85, 3025 (2000).

[8] B. Koopmans, J. J. M. Ruigrok, F. D. Longa, and W. J. M. de Jonge, Phys. Rev. Lett. 95, $267207(2005)$.

[9] J.-Y. Bigot, M. Vomir, and E. Beaurepaire, Nat. Phys. 5, 515 (2009).

[10] J. H. Mentink, J. Hellsvik, D. V. Afanasiev, B. A. Ivanov, A. Kirilyuk, A. V. Kimel, O. Eriksson, M. I. Katsnelson, and T. Rasing, Phys. Rev. Lett. 108, 057202 (2012).

[11] V. López-Flores, N. Bergeard, V. Halté, C. Stamm, N. Pontius, M. Hehn, E. Otero, E. Beaurepaire, and C. Boeglin, Phys. Rev. B 87, 214412 (2013).

[12] A. J. Schellekens and B. Koopmans, Phys. Rev. B 87, 020407 (2013).

[13] C. Boeglin, E. Beaurepaire, V. Halté, V. López-Flores, C. Stamm, N. Pontius, H. Dürr, and J.-Y. Bigot, Nature 465, 458 (2010).

[14] C. Graves, A. Reid, T. Wang, B. Wu, S. de Jong, K. Vahaplar, I. Radu, D. Bernstein, M. Messerschmidt, L. Müller, et al., Nature materials 12, 293 (2013).

[15] B. Vodungbo, J. Gautier, G. Lambert, A. B. Sardinha, M. Lozano, S. Sebban, M. Ducousso, W. Boutu, K. Li, B. Tudu, et al., Nature communications 3, 999 (2012).

[16] I. Radu, G. Woltersdorf, M. Kiessling, A. Melnikov, U. Bovensiepen, J.-U. Thiele, and C. H. Back, Phys. Rev. Lett. 102, 117201 (2009).

[17] I. Radu, K. Vahar, C. Stamm, T. Kachel, N. Pontius, H. Dürr, T. Ostler, J. Barker, R. Evans, R. Chantrell, et al., Nature 472, 205 (2011).

[18] S. Mangin, M. Gottwald, C. Lambert, D. Steil, V. Uhlír, L. Pang, M. Hehn, S. Alebrand, M. Cinchetti, G. Malinowski, et al., Nature materials (2014).

[19] A. Eschenlohr, Element-resolved ultrafast magnetization dynamics in ferromagnetic alloys and multilayers, Ph.D. thesis, Universität Potsdam (2012).

[20] S. Mathias, L.-O. Chan, P. Grychtol, P. Granitzka, E. Turgut, J. M. Shaw, R. Adam, H. T. Nembach, M. E. Siemens, S. Eich, et al., PNAS 109, 4792 (2012).

[21] B. Y. Mueller, A. Baral, S. Vollmar, M. Cinchetti, M. Aeschlimann, H. C. Schneider, and 
B. Rethfeld, Phys. Rev. Lett. 111, 167204 (2013).

[22] L. M. Sandratskii and P. Mavropoulos, Phys. Rev. B 83, 174408 (2011).

[23] I. Radu, C. Stamm, N. Pontius, T. Kachel, P. Ramm, J.-U. Thiele, H. A. Dürr, and C. H. Back, Phys. Rev. B 81, 104415 (2010).

[24] C. Grazioli, C. Callegari, A. Ciavardini, M. Coreno, F. Frassetto, D. Gauthier, D. Golob, R. Ivanov, A. Kivimäki, B. Mahieu, B. Bučar, M. Merhar, P. Miotti, L. Poletto, E. Polo, B. Ressel, C. Spezzani, and G. De Ninno, Rev. Sci. Instrum. 85, 023104 (2014)

[25] X. F. Li, A. L'Huillier, M. Ferray, L. A. Lompré, and G. Mainfray, Phys. Rev. A 39, 5751 (1989).

[26] Z. Chang, A. Rundquist, H. Wang, M. M. Murnane, and H. C. Kapteyn, Phys. Rev. Lett. 79, 2967 (1997).

[27] L. Poletto and F. Frassetto, Appl. Opt. 49, 5465 (2010).

[28] L. Poletto and F. Frassetto, Appl. Sci. 3, 1 (2013).

[29] M. Sacchi, C. Spezzani, P. Torelli, A. Avila, R. Delaunay, and C. F. Hague, Rev. Sci. Instrum. 74, 2791 (2003).

[30] C. La-O-Vorakiat, M. Siemens, M. M. Murnane, H. C. Kapteyn, S. Mathias, M. Aeschlimann, P. Grychtol, R. Adam, C. M. Schneider, J. M. Shaw, H. Nembach, and T. J. Silva, Phys. Rev. Lett. 103, 257402 (2009).

[31] S. Valencia, A. Gaupp, W. Gudat, H.-C. Mertins, P. Oppeneer, D. Abramsohn, and C. Schneider, new Journal of Physics 8, 254 (2006).

[32] C. Stamm, J.-U. Thiele, T. Kachel, I. Radu, P. Ramm, M. Kosuth, J. Minár, H. Ebert, H. A. Dürr, W. Eberhardt, and C. H. Back, Phys. Rev. B 77, 184401 (2008).

[33] C. Bordel, J. Juraszek, D. W. Cooke, C. Baldasseroni, S. Mankovsky, J. Minár, H. Ebert, S. Moyerman, E. E. Fullerton, and F. Hellman, Phys. Rev. Lett. 109, 117201 (2012).

[34] C. Illg, M. Haag, and M. Fähnle, Phys. Rev. B 88, 214404 (2013).

[35] D. W. Cooke, F. Hellman, C. Baldasseroni, C. Bordel, S. Moyerman, and E. E. Fullerton, Phys. Rev. Lett. 109, 255901 (2012).

[36] A. J. Schellekens and B. Koopmans, Phys. Rev. Lett. 110, 217204 (2013).

[37] J. Hohlfeld, S.-S. Wellershoff, J. Güdde, U. Conrad, V. Jähnke, and E. Matthias, J. Chem. Phys 251, 237 (2000).

[38] J. Güdde, J. Hohlfeld, J. Müller, and E. Matthias, Appl. Surf. Sci. 127-129, 40 (1998). 
[39] Z. Lin, L. V. Zhigilei, and V. Celli, Physical Review B 77, 075133 (2008). 